\title{
The spectral composition of fluxes and variances over land and sea out to the mesoscale
}

\author{
Donald H. Lenschow • Jielun Sun
}

Received: 28 July 2006 / Accepted: 24 March 2007 / Published online: 15 June 2007

(C) Springer Science+Business Media B.V. 2007

\begin{abstract}
We discuss the accuracy requirements for measuring mesoscale (roughly horizontal scales $>10 \mathrm{~km}$ or 5 to 10 times the planetary boundary-layer (PBL) depth) fluxes in the convective PBL, and the ability of current research aircraft to achieve this accuracy. We conclude that aircraft equipped with inertial nagivation systems capable of $<3 \mathrm{~km} \mathrm{hr}^{-1}$ navigational accuracy are able to resolve mesoscale fluctuations in velocity, and thus variances and fluxes on the mesoscale. We then discuss measurements of velocity and scalar spectra, and cospectra of vertical velocity with horizontal velocity components and scalars, obtained from long flight legs with the National Center for Atmospheric Research Electra aircraft over the boreal forest of Canada in summer during the BOreal Ecosystem-Atmosphere Study (BOREAS), over the tropical Pacific Ocean from the Tropical Ocean Global Atmosphere Coupled Ocean-Atmosphere Response Experiment (TOGA COARE), and over the East China Sea during wintertime cold-air outbreaks from the Air Mass Transformation Experiment (AMTEX). Each of these studies has somewhat different forcings and boundary conditions, so we can compare their consequences on the spectra and cospectra. On average, we found no significant scalar or momentum fluxes for horizontal scales $>10 \mathrm{~km}$. We also develop a simple model based on observed thermal structure to explain the phase angle between vertical velocity and the along-wind horizontal velocity as a function of height, which shows good agreement with the observed phase angle in AMTEX.
\end{abstract}

Keywords Boundary layer $\cdot$ Flux $\cdot$ Mesoscale $\cdot$ Spectra $\cdot$ Variance

The National Center for Atmospheric Research is sponsored by the National Science Foundation.

D. H. Lenschow $(\bowtie) \cdot J$. Sun

National Center for Atmospheric Research, P.O. Box 3000, Boulder, CO 80307, USA

e-mail: lenschow@ucar.edu 


\section{Introduction}

Measuring contributions to variances and fluxes as functions of wavenumber $k$ in the convective planetary boundary layer (PBL) using instrumented aircraft has become commonplace (e.g.,Lenschow 1986; Oncley et al. 1997). Generally, the spectral region of interest extends from $k$ of order 0.1 cycles $\mathrm{km}^{-1}$ (i.e.scales of several times the depth of the PBL) to about $10^{2}$ cycles $\mathrm{km}^{-1}$. This is the wavenumber region that contains most of the turbulent flux. However, this may not always be the case, as there is no a priori reason for the flux at smaller wavenumbers to always be negligible by comparison. Certainly variances of the velocity components and scalars are not negligible in this mesoscale wavenumber region. Here we consider "mesoscale" as variations on a horizontal scale extending from several times the PBL depth, i.e. $\geq 10 \mathrm{~km}$, to about $100 \mathrm{~km}$. At these small wavenumbers, fixed-point measurements from, e.g., towers cannot adequately resolve the spectral structure since the turbulence time scale is short relative to the advective time scale, and Taylor's "frozen turbulence" hypothesis is no longer valid. In contrast, because an airplane flies more than an order of magnitude faster than the mean wind, its horizontal scale for valid measurements also extends more than an order of magnitude larger.

There are several sources of mesoscale variability that are relevant in this context: first, clouds contained within the PBL or originating at the top of the PBL and extending into the free troposphere can generate mesoscale fluctuations both due to shading of the ground (over land), which modulates the surface energy budget, and dynamical effects resulting from the buoyancy generated by phase changes and by cloud-top radiative cooling. Second, terrain height variations can directly modulate mesoscale flow patterns on the mesoscale over land. Third, variations in surface properties such as surface roughness (e.g., trees vs.grassland) and soil moisture can induce flows that scale with the surface heterogeneity. Finally, dynamical atmospheric processes such as longitudinal rolls, mesoscale cellular convection, and gravity waves in the overlying free troposphere can introduce mesoscale variability directly into the PBL flow field.

The overall effects of these processes on the mesoscale variability of the PBL are hard to assess, partly because of the difficulty in making quantitative observations. As we will show later, the amplitudes of the variations at small $k$ do vary independently of the high wavenumber variations that are driven by surface buoyancy flux and, in the case of scalars, also by surface scalar fluxes.

Here we first consider the limitations on the accuracy of air motion measurements from aircraft at mesoscale wavenumbers, since as far as we know this has not been systematically treated for currently used systems. We assume that an inertial navigation system (INS) is used to obtain the airplane horizontal velocity components and attitude angles. We then show averaged spectra and cospectra from measurements obtained with the NCAR Electra aircraft over the boreal forest of Canada as a contribution to the BOreal Ecosystem-Atmosphere Study (BOREAS) during the summer of 1994, over the western equatorial Pacific Ocean from the Tropical Ocean Global Atmosphere Coupled Ocean-Atmosphere Response Experiment (TOGA COARE) between 15 November 1992 and 17 January 1993 (Sun et al. 1996), and over the East China Sea during the Air Mass Transformation Experiment (AMTEX), which took place in February, 1975. These studies were chosen because they have many long straight flight legs at low levels in the PBL. We do not attempt to normalize the observations with mixed-layer similarity parameters since our focus is on the mesoscale, where appropriate scaling variables are not known, and mixed-layer scaling parameters are not available in all cases. 


\section{Measurement accuracy}

We first consider errors in the vertical air velocity component $w$ measured in the local earth coordinate system as a function of $k$ since its mesoscale fluctuation is considerably smaller than the alongwind $u$ and crosswind $v$ fluctuations due to being constrained by the underlying surface and the capping PBL inversion. We then consider errors in $u$ and $v$. We use wavenumber units of cycles km${ }^{-1}$ instead of $\mathrm{rad} \mathrm{m}^{-1}$ since it is convenient to convert cycles km $\mathrm{k}^{-1}$ to wavelength in $\mathrm{km}$ by taking the reciprocal.

As we show in Fig. 1, the magnitude of mesoscale fluctuations $\left(k<10^{-1}\right.$ cycles km$\left.{ }^{-1}\right)$ in $w$ measured by the Electra in TOGA COARE (which has a smaller fluctuation than BOREAS or AMTEX) is $\sigma_{w m} \simeq 0.1 \pm 0.02 \mathrm{~m} \mathrm{~s}^{-1}$. The statistical significance is based on the conservative assumption that the 31 flight legs used from TOGA COARE translates into 31 degrees-of-freedom for the largest scales We conservatively assume that the minimum signal level needed to accurately measure such a signal is $20 \%$ of this and that the entire contribution to the signal is at $k_{m}=10^{-2}$ cycles $\mathrm{km}^{-1}$, which is close to the minimum observed $k$. The limiting error for mesoscale measurement is likely to be drift in the sensor outputs used to measure $w$. Since the standard deviation of a sine wave of amplitude $A$ is $A / \sqrt{2}$,

$$
\frac{\partial w}{\partial t}<0.2 \sqrt{2} \sigma_{w m} 2 \pi k_{m} \mathcal{U}
$$

This means that for an airplane true airspeed of $\mathcal{U} \simeq 100 \mathrm{~m} \mathrm{~s}^{-1}, \partial w / \partial t \simeq 1.8 \times 10^{-4} \mathrm{~m} \mathrm{~s}^{-2}$. Lenschow (1986) shows that the following approximate relation is adequate for consideration of errors in aircraft $w$ measurements:

$$
w \simeq \mathcal{U} \sin \Theta+w_{p}
$$

where

$$
\Theta \equiv \text { attack angle }- \text { pitch angle }=\alpha-\theta,
$$

and $w_{p}$ is the airplane vertical velocity. The attack angle is the angle of the airflow relative to the aircraft in the vertical plane of the aircraft, positive for upward flow, and the pitch angle is the angle of the longitudinal axis of the aircraft relative to the local horizontal plane of the earth, positive for upward angular displacement of the aircraft nose. Both of these angles are typically $< \pm 5^{\circ}$ for normal research legs, so we make the further approximation in (2) that

$$
w \simeq \mathcal{U} \Theta+w_{p}
$$

For evaluating errors, we differentiate (4) with time and equate it to (1),

$$
\frac{\partial w}{\partial t} \simeq \Theta \frac{\partial \mathcal{U}}{\partial t}+\mathcal{U} \frac{\partial \Theta}{\partial t}+\frac{\partial w_{p}}{\partial t} .
$$

Dynamic pressure from a Pitot tube $q$ is used, along with static pressure $P$ and temperature $T$, to obtain the true airspeed via the relation,

$$
\mathcal{U} \simeq \sqrt{\frac{2 q R T}{P}}
$$

where $R$ is the gas constant. The error in $q$ dominates the error in $\mathcal{U}$. We assume a $q$ measurement accuracy of $0.7 \mathrm{hPa}$ over a nominal $6 \mathrm{~h}$ flight at $100 \mathrm{~m} \mathrm{~s}^{-1}(q \simeq 60 \mathrm{hPa})$ (e.g., Khelif et al. 1999; NCAR, 1991). The value of $\Theta$ will seldom exceed $\pm 2^{\circ}$. Using these values for the first term in (5) gives $\Theta(\partial \mathcal{U} / \partial t) \leq 10^{-6} \mathrm{~ms}^{-2}$. 

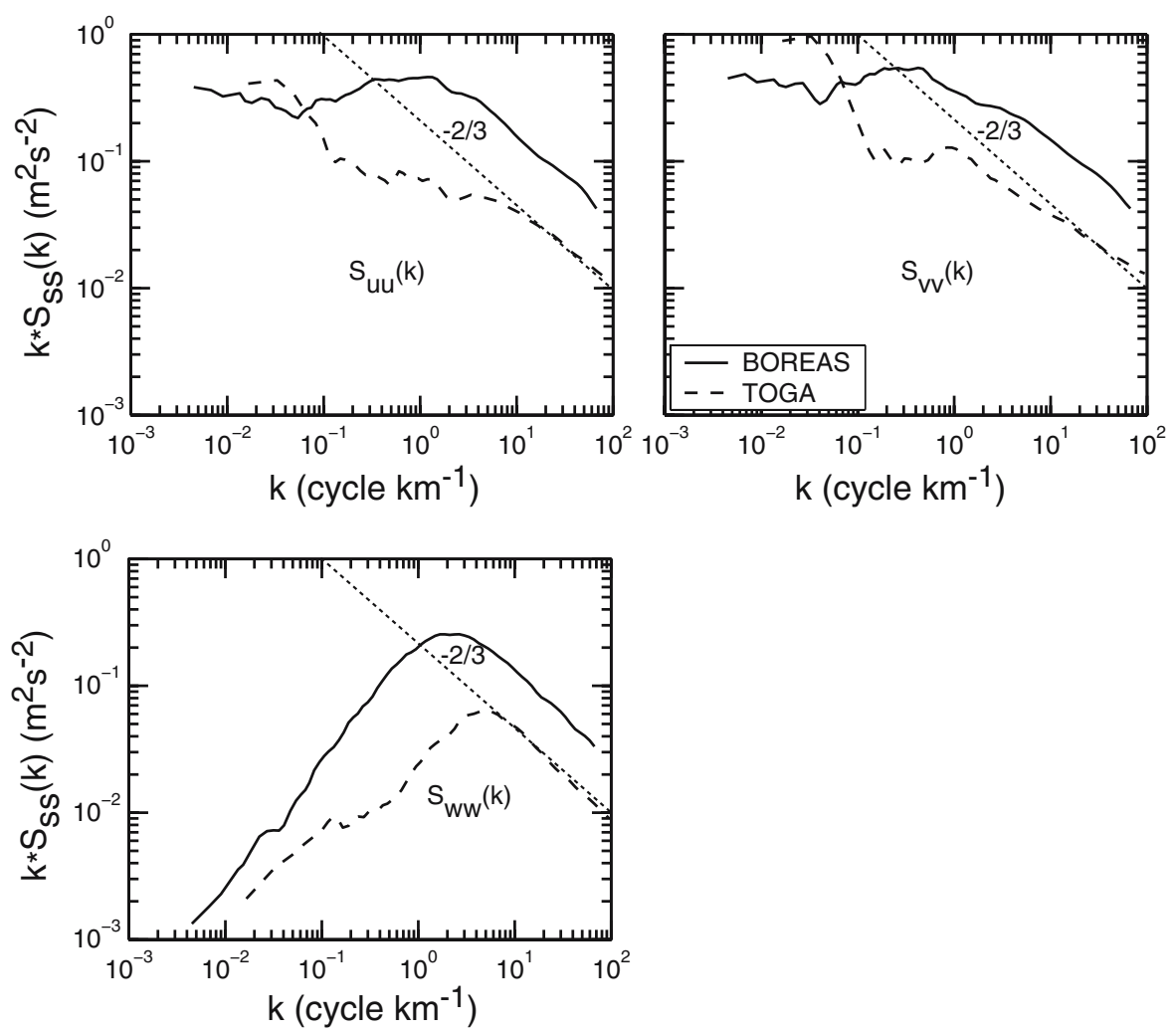

Fig. 1 Spectra of the three velocity components for NCAR Electra flights in BOREAS and TOGA COARE

Considering the second term in (5), the error in $\Theta$ may contain errors in both $\alpha$ and $\theta$, each of which is measured by entirely different technologies. The pitch angle is typically measured with an INS or with an instrument utilizing the Global Positioning System (GPS). The INS maintains an Earth-based coordinate system (either mechanically or computationally) using gyroscopes to maintain an absolute Earth-based reference for the attitude angles of the aircraft, and the doubly integrated output of accelerometers to continuously calculate its position. As the position of the aircraft changes, its angular orientation with respect to the local earth also changes. This means that the INS must continuously update its local orientation so that the gravitational acceleration $g$ is not included in the computation of the updated position from the integrated accelerations. GPS is a satellite-based radio navigation system that measures attitude angles from relative differences in the absolute position of a set of radio receivers mounted some distance apart.

The primary limitation in $\theta$ accuracy from INS is the error contribution from the Schuler oscillation (Britting 1971), whose frequency is given by

$$
\omega_{s}=\sqrt{\frac{g}{R_{e}}},
$$

where $R_{e}$ is the radius of the Earth. This 84.4-min-period oscillation can result from a displacement or perturbation in $\theta$ that introduces an erroneous contribution to the horizontal 
acceleration from $g$. When integrated, this acceleration error rotates the angular reference of the INS in an undamped periodic oscillation about a zero mean.

For an INS with an overall navigational accuracy of $\simeq 3 \mathrm{~km} \mathrm{~h}^{-1}$, the angular stability of the platform, based on the navigation equations of an INS (Britting 1971), is

$$
\frac{\partial \theta}{\partial t}<6 \times 10^{-5} \omega_{s}
$$

which gives $\partial \theta / \partial t \simeq 8 \times 10^{-8} \mathrm{rad} \mathrm{s}^{-1}$. This is in reasonable agreement with the overall $\theta$ accuracy specified for the currently-used Honeywell Laseref SM INS, $\pm 0.05^{\circ}$ over $6 \mathrm{~h}$ duration (NCAR, 2003) if we assume that this error is modulated at the Schuler frequency. Thus, the contribution of $\theta$ to the second term on the right side of (5) is $\mathcal{U}(\partial \theta / \partial t) \leq 8 \times 10^{-6} \mathrm{~m} \mathrm{~s}^{-2}$. Therefore, we conclude that the INS is inherently capable of measuring $\theta$ to sufficient accuracy for measuring mesoscale vertical motions.

Attack angle is measured by means of flow angle sensors mounted on or near the nose of the aircraft. Most commonly, the flow angle is obtained from pressure difference measurements $\delta P$ between a set of ports either located directly on the nose of the aircraft (radome technique; see, e.g., Brown et al. 1983), or on a probe mounted near the front of an aircraft (e.g., Williams et al., 1996) — usually on a boom projecting forward of the aircraft nose (e.g., Khelif et al. 1999). It is difficult to quantify the drift that may occur in this measurement, but we proceed as follows: we use a typical differential pressure sensitivity factor of $\kappa=3.7(\mathrm{rad})^{-1}$ (Brown et al. 1983), where $\kappa$ is defined by the relation

$$
\alpha \equiv \frac{1}{\kappa} \frac{\delta P}{q},
$$

and $q \simeq 60 \mathrm{hPa}$ at $\mathcal{U}=100 \mathrm{~m} \mathrm{~s}^{-1}$. The static pressure transducers used for this measurement by the NCAR Research Aviation Facility (NCAR 1991) have an overall accuracy of $\simeq 0.2 \mathrm{hPa}$; this is mostly due to long-term sources of error such as calibration errors, turnon to turn-on differences, and temperature changes over the entire operating range of the transducers. The contribution of $\alpha$ to the second term on the right side of (5) must satisfy $\mathcal{U}(\partial \alpha / \partial t) \leq 1.8 \times 10^{-4} \mathrm{~m} \mathrm{~s}^{-2}$. Therefore, substituting (9) into the second term of (5) and solving for the pressure drift rate,

$$
\frac{\partial(\delta P)}{\partial t} \leq 1.8 \times 10^{-4} \frac{q \kappa}{\mathcal{U}},
$$

or $\partial(\delta P) / \partial t \leq 4 \times 10^{-4} \mathrm{hPa} \mathrm{s}^{-1}$. Thus, the allowable drift exceeds the long-term accuracy of the $q$ measurement $(0.2 \mathrm{hPa})$ for time periods $>500 \mathrm{~s}$, so we conclude that for mesoscale measurements of $w$, drift in the pressure transducer should not be a problem.

There may, however, be other sources of drift in this term such as changes in mean $\alpha$ due to fuel burn-off or systematic airspeed changes which, combined with undocumented flow distortion effects, may contribute significantly to the drift. For the short-term drift example given above, the allowable drift is only about $0.33 \%$ of the total dynamic pressure. This issue remains an open question worthy of further investigation.

The required vertical acceleration accuracy of $\partial w_{p} / \partial t<1.8 \times 10^{-4} \mathrm{~m} \mathrm{~s}^{-2}$ requires external information, since the vertical acceleration error, in contrast to the horizontal acceleration error, is not constrained within a periodic oscillation. Doubly integrating an erroneous acceleration in the vertical direction leads to a parabolic increase in vertical position error (e.g., Lenschow 1972; Lenschow and Spyers-Duration 1989). Thus, external information is needed to limit vertical position error. $g$ decreases with altitude by about $\partial g / \partial z \simeq-3 \times$ 
$10^{-5} \mathrm{~s}^{-2}$, and to measure $g$ to the required accuracy would require absolute height measurement to $6 \mathrm{~m}$ accuracy over the period of measurement $(\sim 100-1,000 \mathrm{~s})$. This is difficult in practice. Neither standard GPS nor geometric (radar) altitude is generally this accurate (although differential GPS is sufficiently accurate). Furthermore, using geometric altimeters for absolute altitude requires determination of the surface elevation to the same accuracy, which is feasible over the ocean, but difficult over land.

The standard way for measuring height is by means of static pressure measurement. As noted earlier, the static pressure sensor has long-term accuracy of $0.2 \mathrm{hPa}(\simeq 2 \mathrm{~m}$ in height at sea level), but real horizontal pressure variations can be considerably larger than this. To meet the accuracy limit mentioned above of $6 \mathrm{~m}$ over $1,000 \mathrm{~s}$, the horizontal pressure gradient must be $\leq 0.6 \mathrm{~Pa} \mathrm{~km}^{-1}$ (a geostrophic wind of $\simeq 6 \mathrm{~m} \mathrm{~s}^{-1}$ at mid-latitudes), which is often the case in practice, except near strong cyclonic disturbances or deep convection, or in orographic flow.

In contrast to $w$, the small- $k$ spectra (times $k$ ) for $u$ and $v$ are approximately flat (for BOREAS) or increase somewhat (for TOGA COARE) for decreasing $k$ (Fig. 1). Thus, at $k \simeq 10^{-2}$ cycle $\mathrm{km}^{-1}$, the $u, v$ spectra are roughly a factor of 100 larger than the $w$ spectrum, so the accuracy criteria are not nearly so stringent. For convenience in estimating measurement errors, we consider the air velocity measurements in the aircraft coordinate system. We carry out an analysis using the approximate equations presented, e.g., in Lenschow (1986) or Lenschow and Spyers-Duration (1989) (i.e., that cosines of $\alpha, \theta$, and airplane roll angle are unity, terms involving the products of sines of these angles are negligible, and tangents of the flow angles $\simeq$ sines of the flow angles). Rotating the horizontal wind to the aircraft coordinate system,

$$
\begin{aligned}
& u_{x} \simeq-\mathcal{U}+u_{p x} \\
& v_{y} \simeq \mathcal{U} \Psi+v_{p y},
\end{aligned}
$$

where $\left(u_{x}, v_{y}\right)$ are the longitudinal (along the airplane axis) and lateral (normal to the airplane axis) wind components, respectively,

$$
\Psi \equiv \psi^{\prime}+\beta,
$$

where $\psi^{\prime}$ is the departure of the measured true heading from the average true heading and $\beta$ is the sideslip angle (i.e. the airflow angle in the horizontal plane of the aircraft), both measured clockwise looking down from above; and $\left(u_{p x}, v_{p y}\right)$ are the longitudinal (along the airplane axis) and lateral (to the left of the longitudinal aircraft axis looking down) airplane velocity components.

Taking the time derivatives of (11) and (12), we obtain

$$
\begin{aligned}
\frac{\partial u_{x}}{\partial t} & \simeq-\frac{\partial \mathcal{U}}{\partial t}+\frac{\partial u_{p x}}{\partial t} \\
\frac{\partial u_{y}}{\partial t} & \simeq \Psi \frac{\partial \mathcal{U}}{\partial t}+\mathcal{U} \frac{\partial \Psi}{\partial t}+\frac{\partial v_{p y}}{\partial t} .
\end{aligned}
$$

Again considering mesoscale fluctuations; i.e., $k<10^{-2}$ cycles $\mathrm{km}^{-1}$, the standard deviations of the horizontal components (Fig. 1) for both BOREAS and TOGA COARE are $\sigma_{u m} \simeq$ $\sigma_{v m} \simeq 1 \mathrm{~m} \mathrm{~s}^{-1}$. We again conservatively assume that the entire contribution to the mesoscale signal is at $10^{-2}$ cycles $\mathrm{km}^{-1}$ and that we need to measure to $20 \%$ of the measured signal. The required accuracy for mesoscale measurement is $\partial u_{x} / \partial t \simeq \partial v_{y} / \partial t \leq 1.8 \times 10^{-3} \mathrm{~m} \mathrm{~s}^{-2}$.

Evaluating the first term on the right side of (14) using the same Pitot pressure accuracy as was used to estimate the first term in (5), we obtain $\partial \mathcal{U} / \partial t=2.7 \times 10^{-5} \mathrm{~m} \mathrm{~s}^{-2}$. 
The second term in (14) and third term in (15) can be estimated from the expected contribution from the Schuler oscillation (7) of the INS. For a $1 \mathrm{~m} \mathrm{~s}^{-1}$ oscillation, we have $\partial u_{p x} / \partial t \simeq \partial v_{p y} / \partial t \simeq 1.3 \times 10^{-3} \mathrm{~m} \mathrm{~s}^{-2}$. This is also less than required for resolving the observed mesoscale motions, but not by much. However, this can be decreased even more by GPS updating of the horizontal velocity components, which is now routinely done, and was done in BOREAS and TOGA COARE, but not in AMTEX.

The first term in (15) can be evaluated similar to the first term in (5), with the result that $\Psi(\partial \mathcal{U} / \partial t) \leq 10^{-6} \mathrm{~m} \mathrm{~s}^{-2}$. The second term in (15) can be evaluated similar to the second term in (5); however, the INS true heading measurement is less accurate than the $\theta$ measurement since it is not directly constrained by the Schuler oscillation loop. The inherent INS accuracy of currently deployed systems (Britting 1971) gives an acceleration drift rate of $\mathcal{U}\left(\partial \psi^{\prime} / \partial t\right) \leq 10^{-4} \mathrm{~ms}^{-2}$.

Since the sideslip angle is measured with the same technique as $\alpha$, its drift rate is also similar. The accuracy requirement for $u$ and $v$, however, is an order of magnitude less that for $w$, so its contribution to the drift error is proportionately smaller. Thus, all the terms in (14) and (15) are small compared to observed mesoscale horizontal velocity variations.

To summarize, we conclude that the observed spectra of $w$ are within the envelope of the estimated measurement accuracies, and that the $\alpha$ measurement is likely the weakest link. Similarly, the observed $u$ and $v$ spectra are within the envelope of the estimated measurement accuracies, with the true heading drift rate as possibly the weakest link, if GPS is used to update the INS-measured velocity.

\section{Measurement results}

Our data base for measurements over land is from the NCAR Electra flights in the convective PBL that traversed large sections of the boreal forest of Canada during BOREAS. The location and times of the flight legs are given by Oncley et al. (1997), and a brief description of the instrumentation used here by Dobosy et al. (1997). The flights were centered about mid-day throughout the summer, with clear to partly cloudy (mostly fair-weather cumulus) conditions at a height of $\simeq 100 \mathrm{~m}$ above the surface. The 31 legs used here varied from 228 to $666 \mathrm{~km}$ in length (38-111 min). The average height of the PBL was $z_{i} \simeq 1,300 \mathrm{~m}$. The terrain varied from flat to rolling hills, and from solid forest to subarctic tundra, with about $10 \%$ of the tracks over lakes. There is very little evidence of any direct anthropogenic impact on the surface vegetation; the tracks were almost completely north and east of the agricultural limit, and evidence of lumbering was minimal. Therefore, we can consider this dataset as representing atmospheric forcing by interaction with a natural surface unaffected by farming, ranching, lumbering, or urbanization.

We compare these results with measurements from the Electra carried out in the equatorial western Pacific PBL during TOGA COARE. The dataset used here is described by Sun et al. (1996) who previously had partitioned the eddy fluxes into "turbulent," "large-eddy," and "mesoscale" fluxes. Around 26 flight legs ranging between 60 and $120 \mathrm{~km}$ in length and 30 and $40 \mathrm{~m}$ altitude are used here for the analysis. Johnson et al. (2001) estimated an average value of $z_{i}=512 \mathrm{~m}$ from TOGA COARE sounding data. For both BOREAS and TOGA COARE, the horizontal wind components $u, v$ are defined to be alongwind and crosswind, respectively.

We also present observations of the spectral structure of a convective marine boundary layer using Electra measurements during the Air Mass Transformation Experiment (AMTEX) over the East China Sea during February, 1975 (Lenschow and Agee 1976). 
One difference in instrumentation for AMTEX was the use of vanes mounted on a $4 \mathrm{~m}$ nose boom to measure the flow angles (Lenschow et al. 1991); in BOREAS and TOGA COARE, the radome technique (Brown et al. 1983) was used. The time series for all the AMTEX legs considered here extend to over $200 \mathrm{~km}$. In contrast to TOGA COARE, and to most PBLs over the open sea, large surface-air temperature differences occur here due to very cold continental air flowing off the east coast of Asia over a warm, northward flowing ocean current. In this situation a convective baroclinic boundary layer develops which is horizontally homogeneous for turbulence quantities (Lenschow et al. 1980). Furthermore, because the synoptic scale circulation is generally subsiding, there are no deep convective clouds-only stratocumulus of varying degrees of coverage. Details of the measurements are discussed by Lenschow (1986) and Wyngaard et al. (1978).

Out of the total set of constant-level legs flown during AMTEX, four were selected for compositing based on the following criteria:

- Length of the leg (flown at constant level and heading) was at least $2,150 \mathrm{~s}(\simeq 215 \mathrm{~km})$.

- Aircraft heading was within about $\pm 45^{\circ}$ of the line defining the wind direction.

- Height above the surface was $80-100 \mathrm{~m}$.

- Surface radiation temperature was at least $7 \mathrm{~K}$ greater than the potential temperature at flight level.

The height of the inversion at the top of the boundary layer on these days was $1,000 \mathrm{~m}$ $<z_{i}<1,850 \mathrm{~m}$. All of these flights occurred on days with pronounced advection of cold continental air from eastern Asia. Thus, this set of four legs provides a dataset for looking at mesoscale variations in a convective boundary layer over a uniform surface.

Spectral and cospectral quantities were calculated for all the datasets discussed above after removing a linear trend by a least squares fit. In contrast to BOREAS and TOGA COARE, the wind components available from AMTEX are relative to the aircraft axes; that is, we use $u_{x}, v_{y}$. At this point, it is likely impossible to resurrect the AMTEX dataset and redo the calculations in a wind-based coordinate system. However, since the flight legs are aligned approximately along the wind direction, $u_{x}$ and $v_{y}$ are nearly alongwind and crosswind.

One thing to note in comparing these cases is that there is some variability in the airplane height and $z_{i}$, and thus in $z / z_{i}$. As noted in Kaimal and Finnigan (1994), there is little variation in the $u$ and $v$ spectra with height, but the $w$ spectra do show some variation, with the spectral wavenumber peak decreasing somewhat with height up to about $0.2 z_{i}$ where it approaches a mixed-layer form that shows little variation with height.

The AMTEX data in Fig. 2, which contains spectral averages over four legs, each from different flights, indicate that the $u_{x}$ spectrum is about half as large as the $v_{y}$ and $w$ spectra in the inertial subrange. The predicted ratio between the longitudinal and transverse spectra in the inertial subrange is $3 / 4$. Lenschow et al. (1980) have concluded that the $v_{y}$ and $w$ spectra in the inertial subrange give about the right value of dissipation to balance the turbulence energy budget. Therefore, we suspect that the $u_{x}$ spectra at high wavenumbers are too low. One possible cause of this is water accumulation in the Pitot tube or pressure line, which may slow the response of the $u_{x}$ measurement. In support of this, the inertial subrange $u_{x}$ spectra on the first flight are about 3/4 the $v_{y}$ and $w$ inertial subrange spectra; on subsequent flights the spectral ratio is closer to $1 / 2$, but the mean values of $u_{x}$ are not affected. We suspect that water may have accumulated in the tubing sometime during the first flight while penetrating clouds. This may also increase the phase angle between $u_{x}$ and $w$ at large $k$. Fig. 3 shows that the $u_{x}, w$ phase angle does decrease at large $k$. However, this effect does not appear to be very significant for $k<3$ cycles $\mathrm{km}^{-1}$ since the phase angle from the first flight agrees well with the phase angle shown in Fig. 3 for $k$ less than this. (For $k$ greater than this the phase angle 

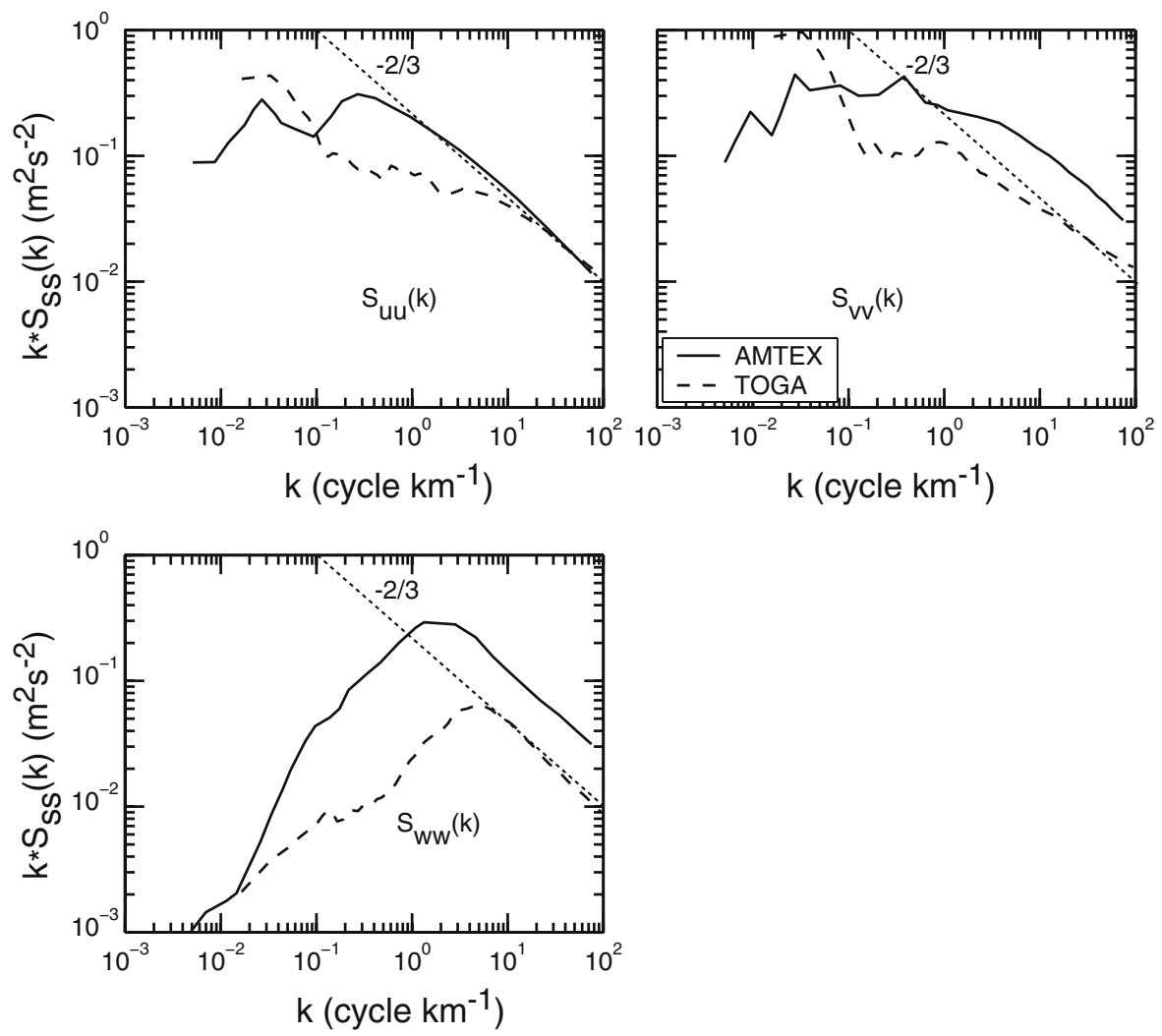

Fig. 2 Spectra of the three velocity components for NCAR Electra flights in TOGA COARE and AMTEX

estimates from Flight 1 have too much scatter.) We also note that flying on opposite headings can remove this error since $u_{x}$ and $v_{y}$ change sign for opposite airplane flight directions.

If we measure a phase angle $\left(\phi_{1}+\delta\right)$ flying at a heading $\psi_{1}$, where $\phi_{1}$ is the measured phase angle between $w$ and $u_{x}$, and $\delta$ is the phase-angle error, we will measure a phase angle $\left(-\phi_{2}+\delta\right)$ flying at a heading $\left(\pi+\psi_{1}\right)$. This is because changing the heading by $\pi$ is equivalent to changing the sign of the measured phase angle. But $\delta$ is independent of flight direction. Therefore, the average phase angle is obtained from the difference between the two measured angles:

$$
\begin{aligned}
\langle\phi\rangle & =\frac{1}{2}\left[\phi_{1}+\delta-\left(-\phi_{2}+\delta\right)\right] \\
& =\frac{\phi_{1}+\phi_{2}}{2} .
\end{aligned}
$$

This is equivalent to our changing the sign of $u_{x}$ when we fly downwind versus upwind so that the average phase angle we measure is the average in a wind-oriented coordinate system.

Finally, we note that the similarity in systems (with the exception that boom-mounted vanes were used in AMTEX, which should not affect the small- $k$ wind measurements) means that the systematic errors should be similar for the three field studies, which adds confidence to the significance of differences in spectra and cospectra among the three studies. 

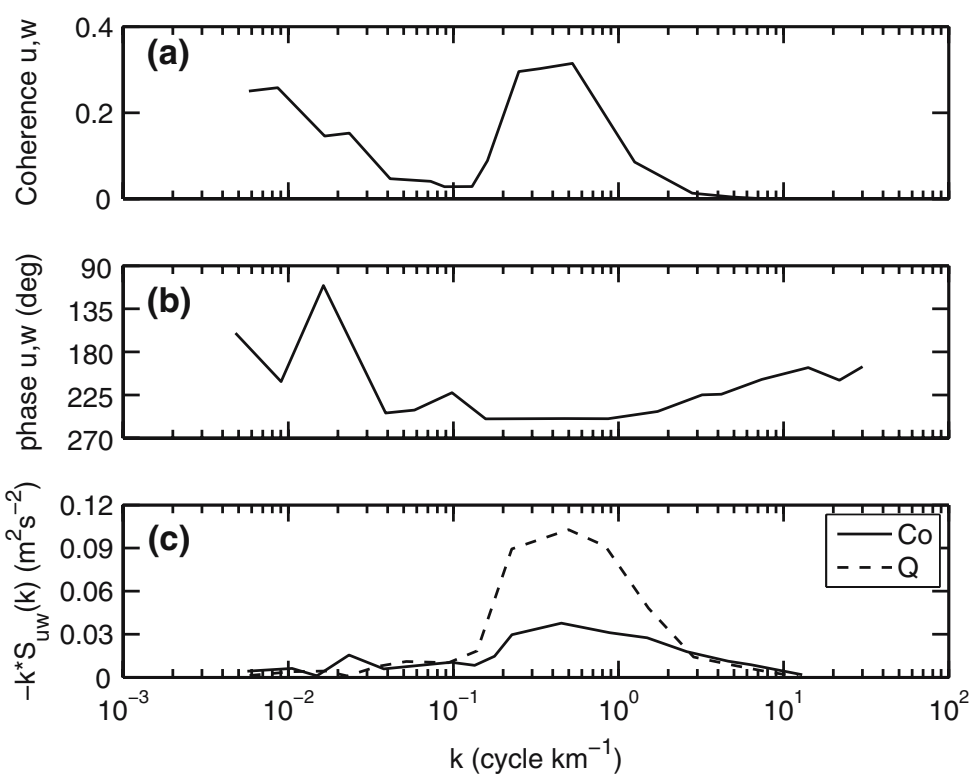

Fig. 3 Coherence, phase, and (negative) cross-spectra of $u_{x}$ and $w$ for the four Electra AMTEX flights considered here

\subsection{Spectra}

We first compare spectra of the three velocity components from BOREAS and TOGA COARE. Fig. 1 shows plots of spectra times $k$. With the exception of the $v$ component in TOGA COARE, the velocity components all have the $-2 / 3$ slope expected in the inertial subrange out to $k$ of nearly 100 cycles $\mathrm{km}^{-1}$, where filtering of the raw data results in a more rapid dropoff with $k$. (Since the horizontal components are defined with respect to the wind direction and the airplane heading was not necessarily along the mean wind, here we do not investigate whether the ratio of the component transverse to the airplane flight track to the component along the track have the predicted $4 / 3$ ratio in the inertial subrange.) The $v$ spectra for TOGA COARE are somewhat flatter than $-2 / 3$, suggesting the possibility of noise at large $k$. At small $k$, i.e., $k$ less than about 1 cycle $\mathrm{km}^{-1}$, the BOREAS $u$ and $v$ spectra flatten out, while the $w$ spectra have a +1 slope. Thus if we had not multiplied the spectra by $k$, the $u$ and $v$ spectra would have had a -1 slope and the $w$ spectra would be flat in this region.

The peak in $k S_{w w}(k)$ is at a smaller $k$ for the BOREAS spectrum than for the TOGA COARE spectrum, as a consequence of both the greater PBL depth and the higher flight level in BOREAS. This also similarly affects the cospectral peaks. The horizontal velocity spectra are known to be little affected by this difference in flight level (Kaimal and Finnigan 1994).

In the inertial subrange, the TOGA COARE spectra are a factor of about 3 smaller than the BOREAS spectra. This is a direct result of the considerably smaller surface buoyancy flux over the ocean. However, the $u$ and $v$ spectra over the ocean crossover at $k \simeq 10^{-1}$ cycles km ${ }^{-1}$. That is, at very small $k$, there is more variability in the horizontal wind field over the ocean than over land. At first glance this seems surprising, since the land surface is more heterogeneous. This suggests that large-scale moist convective processes occurring over the tropical ocean may generate mesoscale variance, in contrast to the temperate PBL over land. 
Thus the relatively large contributions at smaller $k$ may result not so much from horizontal heterogeneity at the surface as from mesoscale processes in the atmosphere.

Boundary-layer scaling is expected to apply in the region around $k=z_{i}^{-1} \simeq 1 \mathrm{cycle} \mathrm{km}^{-1}$. That is, we expect that turbulence energy generated by surface buoyancy flux over a horizontally homogeneous surface should occur in this region, and that the turbulence at larger $k$ is due to the cascade of turbulence from the energy-containing range to the inertial subrange. This coincides with the region where the velocity spectra start to depart from their small- $k$ slopes. Thus comparing the contributions at smaller $k$ to those around $k=z_{i}^{-1} \simeq 1$ cycle $\mathrm{km}^{-1}$ gives us a means of estimating the relative contributions of mesoscale variations to those that scale with $z_{i}$.

Figure 2 is a comparison of velocity spectra from TOGA COARE and AMTEX. We note again that the AMTEX $u$ spectrum is about half as large as the $v$ and $w$ spectra in the inertial subrange, likely due to water accumulation. At large $k$, the AMTEX spectra reflect the large surface buoyancy flux, and their magnitudes are only slightly less than the BOREAS spectra. At small $k$, the AMTEX spectra are slightly less than both the TOGA COARE and the BOREAS spectra, and show some tendency to "flatten out" at small $k$. This offers further support to the suggestion that large-scale moist convective processes are responsible for the mesoscale variability seen in the TOGA COARE spectra, since AMTEX is characterized by stably stratified subsiding flow above the PBL, with no nearby occurrence of deep convection. The horizontal velocity spectral shapes differ somewhat from the results of Kaimal et al. (1976), who found that peaks in the $u$ and $v$ spectra occur at $z_{i} k_{m} \simeq 0.8$, where $k_{m}$ is the wavenumber of maximum spectral amplitude. We do find, however, that the small- $k$ region of the $u, v$ spectra in AMTEX differ considerably from day to day. On days with light winds and small air-sea temperature contrast, small- $k$ variations contribute relatively more to the spectra than on days with strong winds and larger air-sea temperature contrast. It is difficult to generalize the behaviour of the small- $k$ region of the $u, v$ spectra other than that for $k<0.7 z_{i}^{-1}$, the turbulence becomes increasingly two-dimensional; that is, the ratio of the horizontal velocity spectral estimates to the vertical becomes large.

We also note that there is no clearly defined spectral gap in these spectra as in "universal spectra" presented by, for example, Van der Hoven (1957) and Fiedler and Panofsky (1970). However, the Van der Hoven (1957) and Fielder and Panofsky (1970) PBL horizontal wind speed spectra are obtained from composites of tower time series for different frequency intervals pieced together. More recently, Yahaya et al. (2003) present a wind speed spectrum from a cup anemometer at $4 \mathrm{~m}$ height over tilled fields that shows a spectral gap; however, their low-frequency peak lies exactly at the minimum of the Van der Hoven spectrum, and their minimum is less pronounced than for the Van der Hoven spectrum.

The spectra presented here have less amplitude at large $k$ and more at small $k$ than those presented by Van der Hoven (1957). If we extend our consideration of $k S_{u u}(k)$ and $k S_{v v}(k)$ to another decade lower wavenumber, $k \simeq 5 \times 10^{-4}$ cycle $\mathrm{km}^{-1}$, we can see from the AMTEX synoptic maps and surface data that the total variance must be $>20 \mathrm{~m}^{2} \mathrm{~s}^{-2}$, which means that the spectra must increase with decreasing $k$, probably by at least a decade, from $k=5 \times 10^{-3}$ cycle $\mathrm{km}^{-1}$ to $5 \times 10^{-4}$ cycle $\mathrm{km}^{-1}$. Despite this, Lenschow and Stankov (1986) were able to obtain integral scales for $u, v$ in AMTEX from the first zero crossing of the autocorrelation functions that scaled with $z_{i}$, although with considerable scatter. This suggests again that there is some flattening of $k S_{u u}(k)$ and $k S_{v v}(k)$ in the mesoscale region, which is evident in Fig. 2. However, as noted by Durand et al. (2000), if $k S_{u u}(k)$ and $k S_{v v}(k)$ do not approach a slope of +1 at small $k$, which they typically find to be the case, the variance, and thus the integral scale depends on the length of the time series. 
Fig. 4 Spectra of temperature, humidity, $\mathrm{CO}_{2}$, and ozone for the Electra flights in BOREAS

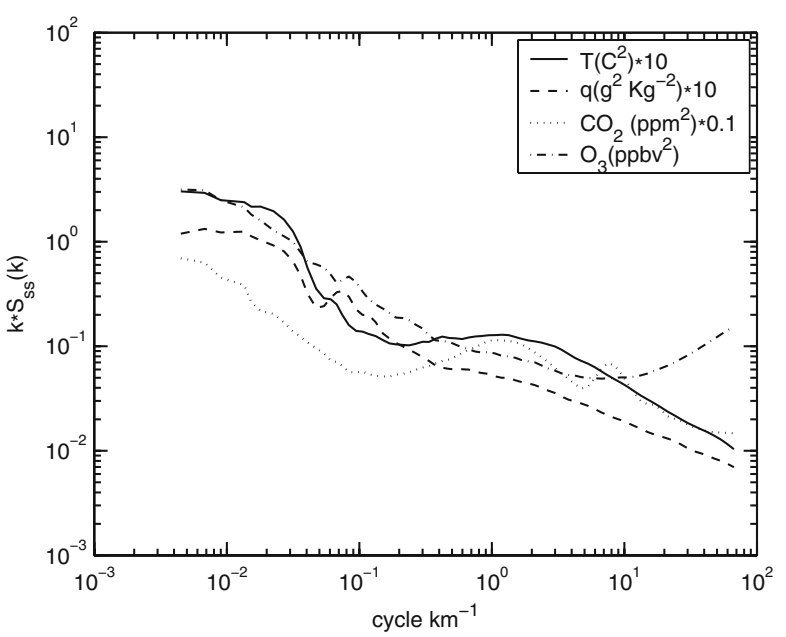

Vertical velocity, by comparison, has a well-defined peak at $k_{m} \simeq 1.7$ cycles km $\mathrm{km}^{-1}$ for both AMTEX and BOREAS, which agrees with the Minnesota results of Kaimal et al. (1976). For $k<2 \times 10^{-2}$ cycles km ${ }^{-1}$, the AMTEX $k S_{w w}(k)$ approaches an approximately constant slope of +1 (equivalently, $S_{w w}(k)$ approaches a constant value). If we assume that this slope continues to $k \rightarrow 0$, the amount of $w$ variance at smaller $k\left(k<0.0046\right.$ cycles km$\left.~^{-1}\right)$ is $\simeq 0.6 \times 10^{-3} \mathrm{~m}^{2} \mathrm{~s}^{-2}$, which is more than three orders of magnitude less than the resolved contributions. Another way of looking at this is to consider that the fluctuations in vertical velocity for wavelengths $>215 \mathrm{~km}$ are about $0.024 \mathrm{~m} \mathrm{~s}^{-1}$. This is about what has been estimated as a typical mean downward velocity over the entire AMTEX array (Sheu and Agee 1977). That is, the independently estimated synoptic-scale $w$ is about the same as the small-wavenumber vertical velocity variance obtained by extrapolating the $w$ spectrum across synoptic scales to $k=0$.

In contrast to the velocity spectra, the scalar spectra from BOREAS, which include temperature $T$, humidity $q$, carbon dioxide, and ozone (Fig. 4), show a flattening in the region around $0.5-5$ cycles $\mathrm{km}^{-1}$, then a sharp rise in magnitude going to small $k$. Thus, the relative contribution of the mesoscale to scalar spectra is considerably larger than for $u$ and $v$ (i.e., somewhat more than a factor of 10 ). We also note that only temperature shows a $-2 / 3$ spectrum in the inertial subrange. The three species measurements contain significant measurement noise in this region, but this does not affect their mesoscale variance (nor their cospectra with $w$ ).

Comparing the BOREAS and TOGA COARE $T$ and $q$ spectra, we see in Fig. 5 that the $T$ spectra over land are about 3-8 times larger than over the tropical ocean in the inertial subrange. This is not surprising based on the considerably larger temperature flux in BOREAS as compared to TOGA COARE. Surprisingly, at small $k$, there is no significant difference between BOREAS and TOGA COARE $T$ spectra. In contrast, the $q$ spectrum in TOGA COARE is about 3-8 times larger than in BOREAS at large $k$, but at small $k$, there is no significant difference. We note, however, that the TOGA COARE $q$ spectrum contains too much noise to make definitive comparisons except at small $k$. In contrast to $u$ and $v, T$ and $q$ spectra do not flatten out for $k \ll 1$ cycles $\mathrm{km}^{-1}$. Instead, they continue to increase by roughly another decade at smaller $k$. That is, these scalar variables have considerably more mesoscale variability, compared to their inertial subrange variance, than $u$ and $v$. 
Fig. 5 Spectra of temperature and humidity for NCAR Electra flights in BOREAS and TOGA COARE

Fig. 6 Spectra of temperature for Electra flights in BOREAS, TOGA COARE, and AMTEX
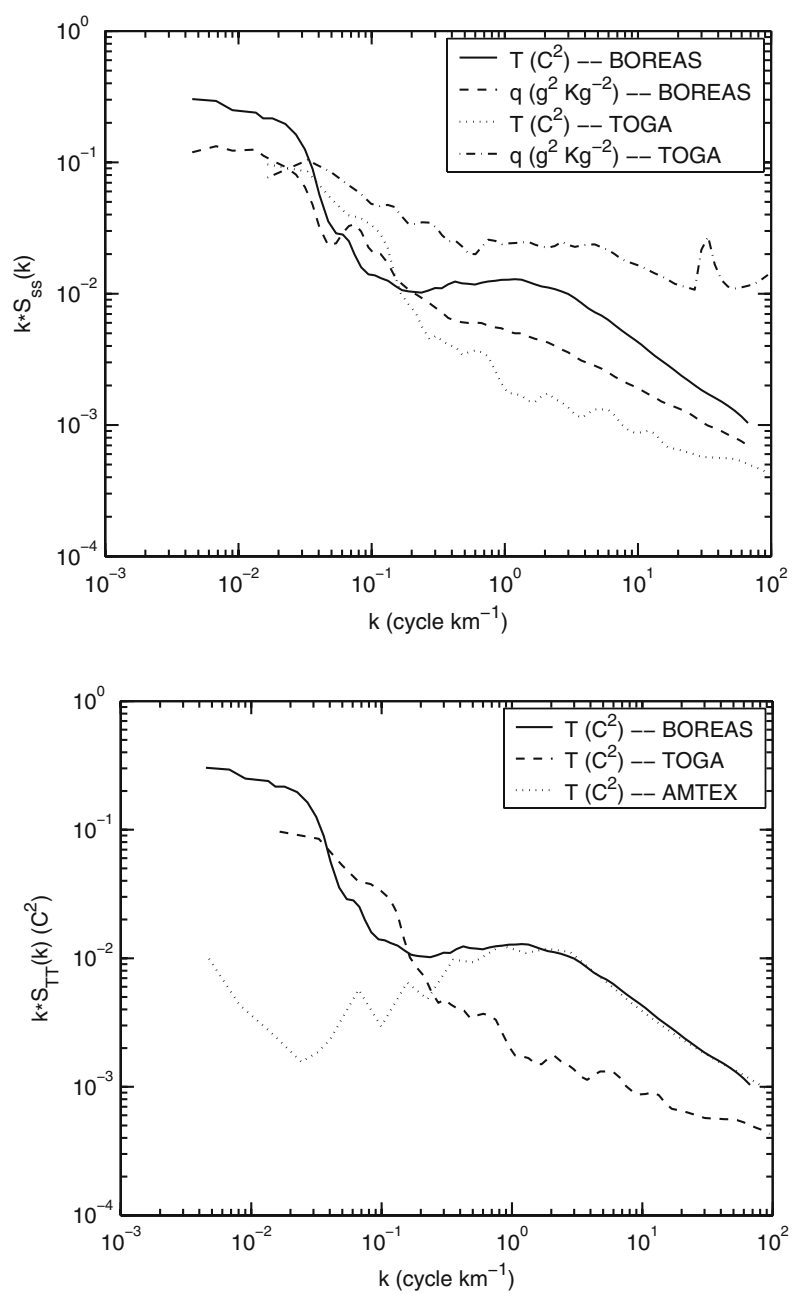

LeMone and Meiten (1984) observed a peak at about $10 \mathrm{~km}$ wavelength in a $q$ spectrum at $150 \mathrm{~m}$ height from an aircraft flying over a similar tropical ocean in the GARP Atlantic tropical Experiment (GATE), but we see no evidence here for such a peak in any of the variables. The $T$ spectrum from BOREAS flattens out in the region $0.1<k<1 \mathrm{~km}^{-1}$, but then increases for smaller $k$, again suggesting that mesoscale temperature fluctuations in BOREAS result from something other than surface temperature flux, although we cannot discount the potential contribution of horizontal variations in surface properties across the boreal landscape.

Fig. 6 shows that the BOREAS and AMTEX $T$ spectra are almost identical at large $k$, but that the AMTEX spectrum changes to a slightly positive slope at small $k$. Thus, the small $k$ region in AMTEX does resemble the $u$ and $v$ spectral shape. We hypothesize that the considerably smaller mesoscale contribution in AMTEX may be the result of an inversion-capped PBL with no penetrative convection. Possibly another reason is the shorter upwind fetch of the AMTEX PBL, which may limit the time for mesoscale fluctuations to develop (de Roode et al. 2004). 
Fig. 7 Cospectra of $w$ and $\bar{\rho} C_{p} T$, and $w$ and $\bar{\rho} L q$, where $C_{p}$ and $L$ are the isobaric specific heat and the latent heat of vaporization for water, respectively, and $\bar{\rho}$ is the mean air density from Electra flights in BOREAS and TOGA COARE

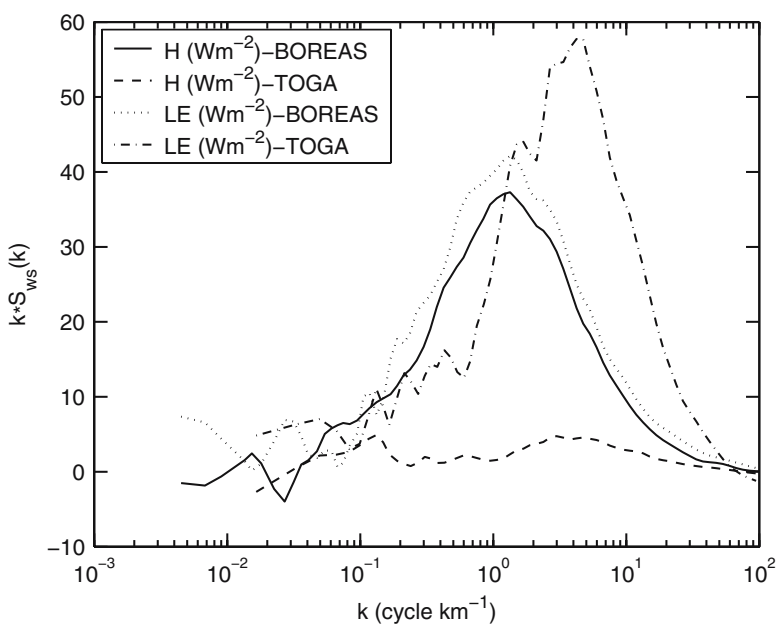

The scalar spectra are consistent with the large-eddy simulation results of de Roode et al. (2004) in the relatively large contribution of the mesoscales to the total variance. They found that the relative contribution to scalar variance at mesoscales is determined by the ratio of entrainment flux to surface flux. They further noted that if the ratio is close to -0.25 , the mesoscale contribution to the variance is minimized. This is consistent with the observation (Fig. 6) that the mesoscale contribution to the $T$ variance is considerably larger for TOGA COARE, where the surface buoyancy flux is mostly due to water vapour-flux (Fig. 7), than for AMTEX, where the $T$ and $q$ fluxes are similar, and the ratio of the entrainment buoyancy flux to the surface buoyancy flux is close to -0.25 (Lenschow et al. 1980). When buoyancy flux is mostly due to water vapour-flux, the entrainment flux of $T$ in a clear-air PBL has a large negative value.

\subsection{Cross-spectral results}

We now examine the cospectra of $w, T, w, q, w, u$, and $w, v$. The $w, T$ and $w, q$ cospectra for BOREAS and TOGA COARE (Fig. 7, which actually shows cospectra of sensible and latent heats for ease of comparison) peak at about the same $k$ as their respective $w$ spectra, and their shapes are very similar. Thus, the differences between the spectra of the scalars and the spectra of $u$ do not lead to any significant differences in their respective cospectra with $w$. That is, the enhanced mesoscale contributions to the scalar spectra do not have a noticeable impact on the cospectra. In other words, on average we do not see significant mesoscale fluxes. LeMone (1978) noted that temperature cospectra from GATE show a sign reversal from positive to negative going from short to long wavelengths, with the negative area becoming increasingly dominant near the top of the PBL as the entrainment flux becomes increasingly important. We see no evidence of this behaviour here, likely because of the low flight level. We also see no evidence of significant fluxes for $k<0.1$ cycle $\mathrm{km}^{-1}$. This means that, on average, mesoscale contributions to the fluxes do not appear to be an explanation of the observation that sometimes both ground-based and aircraft flux measurements seem to underestimate the actual fluxes.

Figure 8 shows that for the BOREAS data, the cospectrum of $w, u$ has a large negative peak at about 1 cycle $\mathrm{km}^{-1}$. The $w, v$ cospectrum has a positive peak at about 3 cycles km $\mathrm{km}^{-1}$. This positive peak is a reflection of the veering, or clockwise rotation, of the wind with height. 

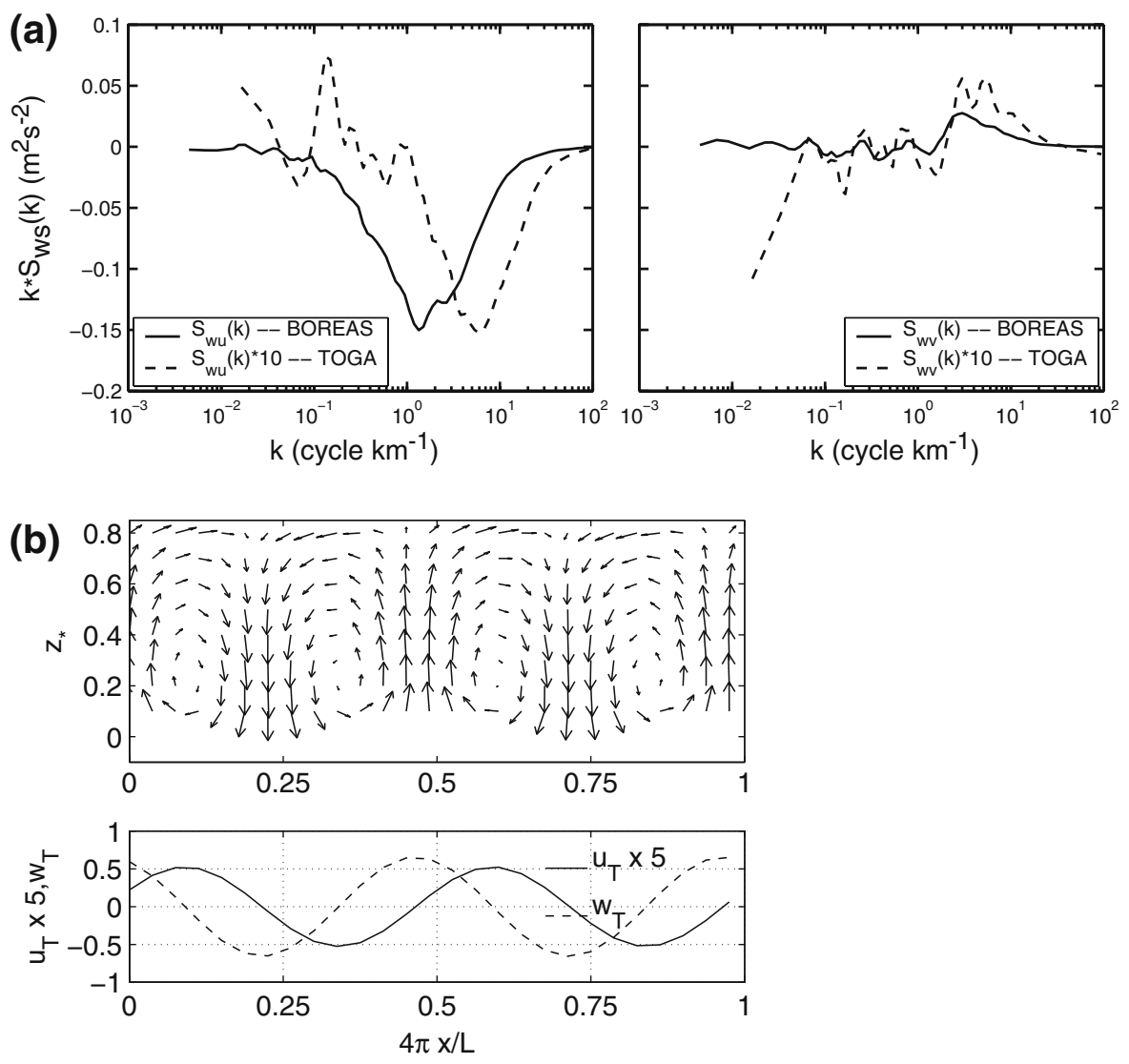

Fig. 8 (a) Cospectra of the two vertical momentum flux components from BOREAS and TOGA COARE. (b) Schematic of momentum transport in a two-dimensional alongwind plane by idealized thermals with the mean wind removed. The thermals are tilted slightly downwind with height because of the downward transport of higher momentum air and upward transport of lower momentum air. The bottom panel shows the corresponding $w$ and $u$ variations at the approximate mid-level of the upper cross-section. The thermal tilt causes an additional phase shift of $\tan ^{-1}\left(\frac{\left\langle u_{T}\right\rangle_{1}}{\left\langle u_{T}\right\rangle_{2}}\right)$ compared to an idealized no-tilt thermal circulation where $w$ lags $u$ by $90^{\circ}$

This means that on average, negative fluctuations of $v$ are transported downward and positive fluctuations upwards. This is consistent with clockwise rotation of the wind with height due to surface friction, so that near the surface $v$ should be greater than higher up. Particularly in the lower part of the PBL, upward transport occurs at smaller scales than downward transport. The higher peak wavenumber for the $w, v$ cospectrum suggests that the upward momentum transport of the $v$ component may be dominant while downward transport of $v$ momentum is either negligible or negative, in contrast to $u$ transport which is likely significant (and the same sign) for both upward and downward transport.

The TOGA COARE momentum cospectra show similar behaviour, but are a factor of 10 smaller, reflecting the smoother surface and generally lighter winds. Furthermore, the peak in the $w, v$ cospectrum is not shifted to larger $k$ as in BOREAS. It is interesting that the ratio 
of the covariances $\langle w u\rangle /\langle w v\rangle$ is about the same for both cases, even though the Coriolis parameter is very much larger for BOREAS than for TOGA COARE.

For the AMTEX data, we have only the $w, u$ cospectral data to work with. Since the AMTEX flight legs were all flown approximately along the mean wind direction, and the mean winds were moderately strong, we looked in detail at the along-axis momentum flux. (For this case, we change the sign of the $u$ component for legs flown upwind so that the phase relation between $u$ and $w$ is the same for both upwind and downwind legs.) Due to the long averaging length, the spectral variables have considerable significance. This encouraged us to study the behaviour of the phase angle between $w$ and $u$, and propose a simple model to explain its behaviour.

Both the (negative) cospecture and quadrature spectra have a peak at about $0.45 \mathrm{cycle} \mathrm{km}^{-1}$, which is about $0.6 z_{i}^{-1}$; however, the cospectrum peak is numerically about $1 / 3$ as large as the quadrature spectrum peak. This means that $w$ lags $u$ by about $108^{\circ}$ (or as shown in Fig. 3 a positive phase angle of $252^{\circ}$ ) for an upwind flight leg. (We define positive $u$ as being along the flight direction, so that on a downwind flight leg, $u$ is negative and thus the phase relationship remains the same.) There is also some indication that the phase angle is less at larger $k$, although this is not very conclusive because of the decreasing significance of the crossspectral estimates and the possible contribution from instrumental lag in the $u$ measurement. Application of (16) results in a reduction of this contribution by a factor of two over what it would be from a single leg, since one leg is downwind while three legs are upwind.

The phase angle between $w$ and $u$ in the lower part of the convective boundary layer differs considerably from the phase between $w$ and scalar variables. Typically the $w, u$ phase angle is somewhat greater than $-90^{\circ}$, so that the momentum flux, $-\left\langle u^{\prime} w^{\prime}\right\rangle>0$, but the quadrature spectrum magnitude is considerably larger than the cospectrum magnitude. On the other hand, the phase angle between $w$ and scalar variables (e.g., $T$ and $q$ ) is normally close to $0^{\circ}$ or $180^{\circ}$; i.e., the quadrature spectrum magnitude is normally considerably less than the cospectrum magnitude.

The behavior of the $w, u$ cross-spectra is more complicated than for scalar cross-spectra because of dynamical character of momentum transport. Furthermore, the $w, u$ coherence is also typically less than $w$, scalar coherence. Therefore, we consider it in more detail using the AMTEX data, for which we have detailed vertical flux profiles for a number of very similar cases, good estimates of $z_{i}$, and a large dataset for good statistical significance.

The cross-spectral behaviour of $w, u$ can be analyzed with a simple model that divides the boundary layer into plumes (or thermals) and their environment. The mean circulation of plumes consists of warm, usually moist air flowing into the plume near the surface, where the updraft accelerates, and out near the top, where the plume decelerates (Lenschow and Stephens 1980). Thus, if there were no wind shear, we would expect that $w$ would lag $u$ by $90^{\circ}$ (a phase shift of $270^{\circ}$ ), and no momentum transport would occur. Since in fact the wind speed goes to zero at the surface, the updraft air, particularly in the lower part of the boundary layer, must have a wind-speed deficit. As a result, phase shift is different from $270^{\circ}$ and momentum is transported by the plume circulation, as shown schematically in Fig. 8b. This is consistent with the observations of Wilczak and Tillman (1980), who noted that the plume translational velocity is less than the mean wind speed at the uppermost height of the plume and greater than the mean wind speed near the surface.

We also note that the character of momentum transport is quite different in the mixed layer, where shear is minimal, compared to the surface layer, where shear is large. Due to the large surface-layer shear, plumes are tilted downstream, as noted by Kaimal and Businger (1970), and Wilczak and Tillman (1980). However, above the surface layer, the absence of shear results in minimal plume tilt, as noted (Lothon et al. 2006). 
The magnitude of the thermal circulation can be estimated from the relation obtained by Lenschow and Stephens (1980) for the average vertical velocity of a field of plumes,

$$
\left\langle w_{T}\right\rangle / w_{*}=z_{*}^{1 / 3}\left(1-1.1 z_{*}\right)
$$

where $w_{*}=\left[(g / T) Q_{o v} z_{i}\right]^{1 / 3}, Q_{o v}$ being the surface virtual temperature flux, $g / T$ the buoyancy parameter and $z_{*}=z / z_{i}$, where $z$ is the height above the surface. They found that the area covered by thermals, as defined by their criteria of humidity exceeding the mean by half a standard deviation, was about $28 \%$. Thus, (17) represents the updraft averaged over $28 \%$ of the total area. We approximate the composite updraft velocity profile with a Fourier series expansion,

$$
w_{T}=\left\langle w_{T}\right\rangle \sum_{m=1}^{\infty} a_{m} \cos \left(2 \pi m x / L+\phi_{m}(z)\right),
$$

where $L$ is the fundamental wavelength of the plume-scale circulation, which is assumed to approximately coincide with the wavelength of the spectral peak, and $\phi_{m}(z)$ is the phase angle of the $m t h$ contribution to the sum. We assume that the shape of the updraft velocity is an even function about its mid-point so that the Fourier expansion contains only cosines, and that the shape does not change with height so that $\phi$ is only a function of height and not of $m$. Then

$$
\begin{aligned}
\frac{\partial w_{T}}{\partial z}= & \frac{\partial\left\langle w_{T}\right\rangle}{\partial z} \sum_{m=1}^{\infty} a_{m} \cos (2 \pi m x / L+\phi) \\
& -\left\langle w_{T}\right\rangle \frac{\partial \phi}{\partial z} \sum_{m=1}^{\infty} a_{m} \sin (2 \pi m x / L+\phi) .
\end{aligned}
$$

Substituting (17) into (19),

$$
\begin{aligned}
\frac{\partial w_{T}}{\partial z}= & w_{*} z_{*}^{1 / 3}\left[\frac{\left(1-4.4 z_{*}\right)}{3 z} \sum_{m=1}^{\infty} a_{m} \cos (2 \pi m x / L+\phi)\right. \\
& \left.-\left(1-1.1 z_{*}\right) \frac{\partial \phi}{\partial z} \sum_{m=1}^{\infty} a_{m} \sin (2 \pi m x / L+\phi)\right] .
\end{aligned}
$$

We assume that horizontal variations in $u$ and $v$ are also sinusoidal, and that the composite thermal shape is axially symmetric so that on average we can assume two-dimensionality. Then, assuming incompressibility and steady-state conditions, by continuity we obtain

$$
\frac{\partial u_{T}}{\partial x}=-\frac{1}{2} \frac{\partial w_{T}}{\partial z} .
$$


Substituting (20) into (21) and integrating along the flight path,

$$
\begin{aligned}
u_{T}= & u_{T 1}+u_{T 2} \\
= & \left\langle u_{T}\right\rangle_{1} \sum_{m=1}^{\infty} \frac{a_{m}}{m} \sin (2 \pi m x / L+\phi) \\
& +\left\langle u_{T}\right\rangle_{2} \sum_{m=1}^{\infty} \frac{a_{m}}{m} \cos (2 \pi m x / L+\phi), \\
= & \sum_{m=1}^{\infty} \frac{a_{m}}{m} \cos \left(2 \pi m x / L+\phi-\phi_{u w}\right)
\end{aligned}
$$

where

$$
\begin{aligned}
\left\langle u_{T}\right\rangle_{1} & =-\frac{w_{*} z_{*}^{1 / 3} L\left(1-4.4 z_{*}\right)}{12 \pi z}, \\
\left\langle u_{T}\right\rangle_{2} & =-\frac{w_{*} z_{*}^{1 / 3} L\left(1-1.1 z_{*}\right)}{4 \pi} \frac{\partial \phi}{\partial z}, \\
\phi_{u w} & =\tan ^{-1}\left[\frac{\left\langle u_{T}\right\rangle_{1}}{\left\langle u_{T}\right\rangle_{2}}\right] .
\end{aligned}
$$

Equations (24) and (25) are the amplitudes of the components that are out-of-phase and inphase, respectively, with $w_{T}$, and $\phi_{u w}$ is the phase angle by which $w_{T}$ lags $u_{T}$. By Parseval's theorem, the covariance, which is the thermal-scale momentum flux, can be written as $1 / 2$ the sum of the products of the series coefficients:

$$
\left\langle u_{T 2} w_{T}\right\rangle=\frac{\left\langle u_{T}\right\rangle_{2}\left\langle w_{T}\right\rangle}{2} \sum_{m=1}^{\infty} a_{m}^{2} / m .
$$

The normalized covariance of a field of thermals is characterized by a "top hat" shape covering $28 \%$ of the total record length. The Fourier coefficients of this top-hat shape can be used to evaluate the summation in (27), which gives $\sum_{m=1}^{\infty} a_{m}^{2} / m \simeq 0.58$. Therefore,

$$
\left\langle u_{T}\right\rangle_{2}=3.4 \frac{\left\langle u_{T 2} w_{T}\right\rangle}{\left\langle w_{T}\right\rangle} .
$$

Solving (26) for $\left\langle u_{T}\right\rangle_{2}$, substituting this and (17) into (28), assuming $L=1.3 z_{i}$ (Kaimal et al. 1976), and solving for the thermal-scale momentum flux,

$$
\left\langle u_{T 2} w_{T}\right\rangle \simeq-0.01 \frac{w_{*}^{2}\left(1-4.4 z_{*}\right)\left(1-1.1 z_{*}\right)}{z_{*}^{1 / 3} \tan \phi_{u w}} .
$$

Lenschow et al. (1980) show that for $z_{*}<0.5$ in the baroclinic AMTEX PBL,

$$
\left\langle u^{\prime} w^{\prime}\right\rangle \simeq-u_{*}^{2}\left(1-2 z_{*}\right) .
$$

$u_{*}^{2}$ was measured to be about $0.20 \mathrm{~m}^{2} \mathrm{~s}^{-2}$, and thus from (30), $\left\langle u^{\prime} w^{\prime}\right\rangle \simeq-0.17 \mathrm{~m}^{2} \mathrm{~s}^{-2}$ at aircraft height $\left(z \simeq 0.073 z_{i} \simeq 100 \mathrm{~m}\right)$. For the AMTEX data we present in Fig. 3, $z_{*}=0.073$, $w_{*}=1.84 \mathrm{~m} \mathrm{~s}^{-1}$ and, for the thermal-scale mode $\left(0.1<k<1\right.$ cycles km$\left.{ }^{-1}\right), \phi_{u w} \simeq 252^{\circ}$. Substituting these values into (29), we obtain $\left\langle u_{T 2} w_{T}\right\rangle \simeq-0.017 \mathrm{~m}^{2} \mathrm{~s}^{-2}$. We assume that $\left\langle u_{T 2} w_{T}\right\rangle$ is proportional to the total momentum flux $\left\langle u^{\prime} w^{\prime}\right\rangle$ throughout the entire PBL. Then, from the above values,

$$
\left\langle u_{T 2} w_{T}\right\rangle /\left\langle u^{\prime} w^{\prime}\right\rangle \simeq 0.1
$$




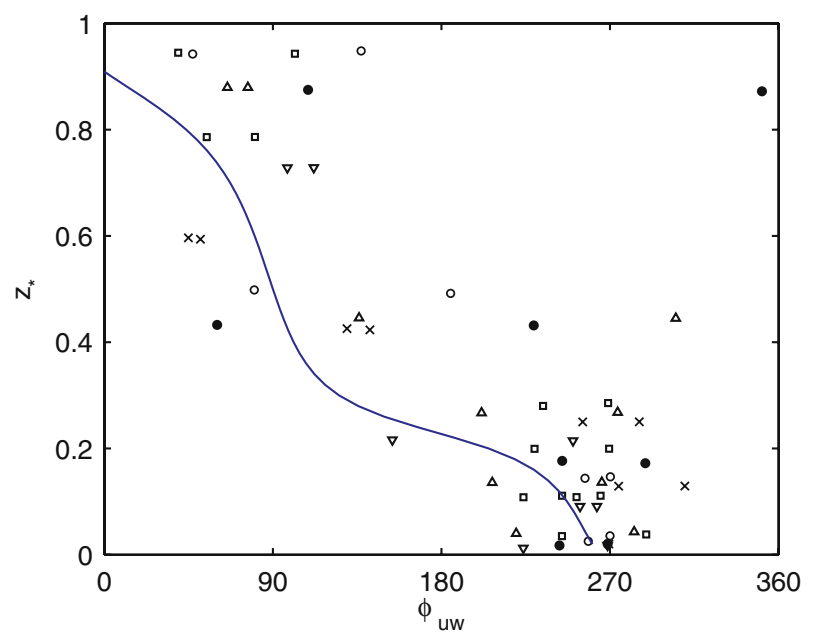

Fig. 9 Plot of phase angle (degrees) between $w$ and $u$ versus normalized height for all the sets of Electra flight legs from AMTEX used in the analysis of mixed-layer structure: $\square=15 \mathrm{Feb} ; \nabla=16 \mathrm{Feb} ; \bullet=18 \mathrm{Feb}$; $\times=22 \mathrm{Feb} ; \circ=24 \mathrm{Feb} ; \triangle=26 \mathrm{Feb}$

Substituting (17), (24), (28), (30), (31), and $L=1.3 z_{i}$ into (26), we have

$$
\begin{aligned}
\phi_{u w} & =\tan ^{-1}\left[\frac{-0.1\left(w_{*} / u_{*}\right)^{2} z_{*}^{-1 / 3}\left(1-4.4 z_{*}\right)\left(1-1.1 z_{*}\right)}{-\left(1-2 z_{*}\right)}\right] \\
& =\pi+\tan ^{-1}\left[\frac{0.1\left(w_{*} / u_{*}\right)^{2} z_{*}^{-1 / 3}\left(1-4.4 z_{*}\right)\left(1-1.1 z_{*}\right)}{\left(1-2 z_{*}\right)}\right]
\end{aligned}
$$

This function is plotted in Fig. 9 for the values of $z_{*}, u_{*}$, and $w_{*}$ that characterized the AMTEX PBL using the same set of flight legs that Lenschow et al. (1980) used in their analysis of mixed-layer turbulence structure. Also plotted in this figure are observations of the phase angle throughout the AMTEX boundary layer from this set of flight legs. The points are generally in agreement with the theoretical curve.

We note that (33) predicts that the phase angle approaches $3 \pi / 2$ near the surface, then decreases with height. At $z_{*} \simeq 0.23, u$ and $w$ are in antiphase. The phase angle continues to decrease with height above this level, and may again become in phase in the upper part of the boundary layer. The antiphase level at $z_{*}=0.23$ is independent of $u_{*}$ and $w_{*}$. Thus, it may be possible to estimate $z_{i}$ by measuring only the $w, u$ phase angle through the lower $1 / 4$ of the convective boundary layer.

We can also calculate the phase angle of thermals as a function of height. Solving (25) for $z_{i}(\partial \phi / \partial z)=\partial \phi / \partial z_{*}$, substituting (28), (30), (31), and (17) into the expression, and integrating yields

$$
\phi\left(z_{*}\right)-\phi(0)=\int_{0}^{z_{*}} \frac{3.3\left(u_{*} / w_{*}\right)^{2}\left(1-2 z_{*}^{\prime}\right)}{\left(1-1.1 z_{*}^{\prime}\right)^{2} z_{*}^{\prime 2 / 3}} d z_{*}^{\prime} .
$$

Figure 10 shows a profile of $\phi\left(z_{*}\right)-\phi(0)$, which is the phase angle of the thermal at $z_{*}$ relative to the phase angle at the surface. We see that in the middle of the PBL, there is little variation with height; e.g. for $0.2 \leq z_{*} \leq 0.7$ the change is less than $8^{\circ}$. This is consistent 
Fig. 10 Plot of the phase angle of thermals as a function of normalized height $z_{*}$ relative to its value at the surface

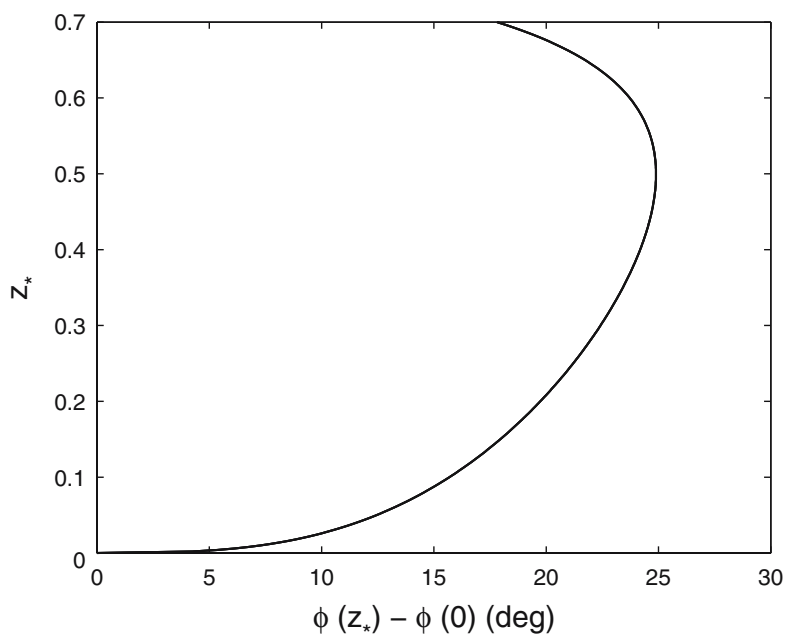

with the results of Lothon et al. (2006), who found no discernable phase shift of $w$ with height throughout the middle of the convective PBL.

\section{Conclusions}

We have shown that currently used INS-based air motion systems on, e.g., the NCAR Electra aircraft can measure mesoscale variations (out to $k<10^{-2}$ cycles km${ }^{-1}$ ) of the three wind components in the convective PBL over both land and sea. We then present summary spectra and cospectra from experiments over the boreal forest of Canada (BOREAS) in summer, the equatorial western Pacific Ocean (TOGA COARE), and the East China Sea during wintertime cold air outbreaks (AMTEX). The land measurements were obtained at a height of $\simeq 100 \mathrm{~m}$ above the surface, the tropical ocean measurements at a height of $30-40 \mathrm{~m}$, and the cold air outbreak measurements at $80-100 \mathrm{~m}$. The results provide a basis for specifying typical levels of scale-resolved turbulence and flux contributions over land and sea.

Over land, $k S_{w, u, v}(k)$ are about three times larger than over the tropical ocean in the inertial subrange (where the spectra display a $-2 / 3$ slope). Furthermore, $k S_{w}(k)$ shows a

$k^{-1}$ dependency at mesoscales $\left(4 \times 10^{-3}<k<10^{-1}\right)$ (i.e., a flat $S_{w}(k)$ spectrum) while $k S_{u, v}$ are flat. Over the tropical ocean, the mesoscale values of $S_{w, u, v}(k)$ relative to the inertial subrange are larger. This means that the spectra from the two regimes are not similar in shape, and that the mesoscale fluctuations, at least over the relatively homogeneous ocean, result from mesoscale processes rather than local turbulence generation by buoyancy and shear. The cold air outbreak measurements have the least mesoscale energy-possibly due to the absence of penetrative convection.

Cospectra of the vertical fluxes of both scalars and momentum show that on average the mesoscale contributions $\left(k<10^{-1}\right.$ cycles $\left.\mathrm{km}^{-1}\right)$ are insignificant. Of course, this does not mean that the mesoscale fluxes for individual cases are insignificant, only that on average they seem to cancel out. This is consistent with Sun et al. (1996).

We also present a simple model, based on observed thermal velocity structure, to account for the observed phase shift between $w$ and $u$ throughout the convective PBL. The predicted phase shift, which decreases from $\simeq 252^{\circ}$ near the surface to $\simeq 0^{\circ}$ at $z_{*} \simeq 0.9$, agrees well 
with that observed in AMTEX. The model predicts minimal tilt to the thermals in the middle of the PBL, as is indicated by observations (Lothon et al. 2006).

These results provide useful guidance for estimating spectra of the three velocity components and scalars, and cospectra of these variables with vertical velocity in the convective boundary layer over both land and ocean.

Acknowledgements We thank the staff of the NCAR Research Aviation Facility who provided the tools to carry out this research. Alastair Williams gave us information on the TOGA COARE dataset. Leif Kristensen provided invaluable insight and assistance with the modelling of the phase angle between $w_{T}$ and $u_{T}$. We thank Marie Lothon and an anonymous reviewer for their helpful comments.

\section{References}

Britting KR (1971) Inertial navigation systems analysis. Wiley, New York, 249 pp

Brown EN, Friehe CA, Lenschow DH(1983) The use of pressure flctuations on the nose of an aircraft for measuring air motion. J Appl Meteorol 22:171-180

de Roode SR, Duynkerke PG, Jonker HJJ(2004) Large-eddy simulation: how large is large enough. J Atmos Sci 61:403-421

Dobosy RJ, Crawford TL, MacPherson JI, Desjardins RL, Kelly R, Oncley SP, Lenschow D(1997) Intercomparison among the four flux aircraft at BOREAS in 1994. J Geophys Res 102:29101-29111

Durand P, Thoumieux F, Lambert D (2000) Turbulent length-scales in the marine atmospheric mixed layer. Quart J Roy Meteorol Soc 126:1889-1912

Fiedler F, Panofsky HA (1970) Atmospheric scales and spectral gaps. Bull Am Meteor Soc 51:1114-1119

Johnson RH, Ciesielski PE, Cotturone JA (2001) Multiscale variability of the atmospheric mixed layer over the western Pacific warm pool. J Atmos Sci 58:2729-2750

Kaimal JC, Businger JA (1970) Case studies of a convective plume and a dust devil. J Appl Meteorol 9:612-620

Kaimal JC, Finnigan JJ (1994) Atmospheric boundary layer flows: their structure and measurement. Oxford University Press, New York, 289 pp

Kaimal JC, Wyngaard JC, Haugen DA, Coté OR, Izumi Y(1976) Turbulence structure in the convective boundary layer. J Atmos Sci 33:2152-2169

Khelif D, Burns SP, Friehe CA (1999) Improved wind measurements on research aircraft. J Atmos Oceanic Technol 16:860-875

Lemone MA (1978) The marine boundary layer. In: Wyngaard JC (ed) Workshop on the planetary boundary layer. American Meteorological Society, 45 Beacon St, Boston, MA, pp 182-234

LeMone MA, Meitin RJ(1984) Three examples of fair-weather mesoscale boundary layer convection in the tropics. Mon Wea Rev 112:1985-1997

Lenschow DH (1972) The measurement of air velocity and temperature using the NCAR Buffalo aircraft measuring system. Technical Report NCAR-TN/EDD-74, National Center for Atmospheric Research, $39 \mathrm{pp}$

Lenschow DH (1986) Aircraft measurements in the boundary layer. In: Lenschow DH (ed) Probing the atmospheric boundary layer. American Meteorological Society, Boston, MA, pp 39-55

Lenschow DH, Agee EM(1976) Preliminary results from the Air Mass Transformation Experiment (AMTEX). Bull Am Meteor Soc 57:1346-1355

Lenschow DH, Spyers-Duran P (1989) Measurement techniques: air motion sensing. National Center for Atmospheric Research, Bulletin No. 23, available at: http://raf.atd.ucar.edu/Bulletins/bulletin23.html

Lenschow DH, Stankov BB (1986) Length scales in the convective boundary layer. J Atmos Sci 43:1198-1209

Lenschow DH, Stephens PL (1980) The role of thermals in the convective boundary layer. Boundary-Layer Meteorol 19:509-532

Lenschow DH, Wyngaard JC, Pennell WT(1980) Mean-field and second-moment budgets in a baroclinic, convective boundary layer. J Atmos Sci 37:1313-1326

Lenschow DH, Miller ER, Friesen RB(1991) A three-aircraft intercomparison of two types of air motion measurement systmes. J Atmos Oceanic Technol 8:41-50

Lothon M, Lenschow DH, Mayor SD (2006) Coherence and scale of vertical velocity in the convective boundary layer from a Doppler lidar. Boundary-Layer Meteorol 121:521-536

NCAR (1991) Pressure measurement from NCAR Aircraft. National Center for Atmospheric Research, Bulletin No. 21, available on the web at: http://www.eol.ucar.edu/raf/Bulletins/bulletin21.html 
NCAR (2003) The NSF/NCAR C-130Q Hercules (N130AR): overview and summary of capabilities. National Center for Atmospheric Research, Bulletin No. 3, available on the web at: http://www.eol.ucar.edu/raf/Bulletins/bulletin3.html

Oncley SP, Lenschow DH, Davis KJ, Campos TL(1997) Regional-scale surface flux observations across the boreal forest during BOREAS. J Geophys Res 102:29147-29154

Sheu P-J, Agee EM(1977) Kinematic analysis and air-sea heat flux associated with mesoscale cellular convection during AMTEX-75. J Atmos Sci 34:793-801

Sun J, Howell JF, Esbensen SK, Mahrt L, Greb CM, Grossman R, LeMone MA(1996) Scale dependence of air-sea fluxes over the western Equatorial Pacific. J Atmos Sci 53:2997-3012

Van der Hoven I(1957) Power spectrum of horizontal wind speed in the frequency range from 0.0007 to 900 cycles per hour. J Meteorol 14:160-164

Wilczak JM, Tillman JE(1980) Three-dimensional structure of convection in the atmospheric surface layer. J Atmos Sci 37:2424-2443

Williams A, Kraus H, Hacker J(1996) Transport processes in the tropical warm pool boundary layer. Part I: Spectral composition of fluxes. J Atmos Sci 53:1187-1202

Wyngaard JC, Pennell WT, Lenschow DH, LeMone MA (1978) The temperature-humidity covariance budget in the convective boundary layer. J Atmos Sci 35:47-58

Yahaya S, Frangi JP, Richard DC (2003) Turbulent characteristics of a semiarid atmospheric surface layer from cup anemometers - effects of soil tillage treatment (Northern Spain). Ann Geophysicaie 21:2119-2131 